\title{
L'implantation de tissu pancréatique endocrine
}

La greffe de pancréas, chez des malades diabétiques, reste une intervention majeure, qui s'applique surtout en association à une transplantation rénale. L'implantation de tissu insulaire apparaît, dans son principe, comme une alternative intéressante dont le succès peut être modulé par les paramètres suivants : la quantité de cellules $\beta$ disponibles ; la localisation de l'implant et ses effets sur les réactions immunitaires et la fonctionnalité du greffon; les statuts immunologiques du receveur et du donneur ; la réduction de l'alloréactivité des greffes par des prétraitements comme l'encapsulation, la purification des cellules $\beta$ ou la destruction des leucocytes passagers. A ce jour, il reste encore à prouver que les greffes de tissu insulaire peuvent rétablir à long terme un contrôle du métabolisme glucidique supérieur à celui obtenu par l'insulinothérapie et éviter, mieux que les traitements classiques, la survenue des complications chroniques.

\section{Danny Pipeleers}

ADRESSE

D. Pipeleers : docteur en médecine, docteur ès sciences. Département de métabolisme et endocrinologie, Vrije Universiteit Brussel Laarbeeklaan 103, B-1090 Bruxelles,

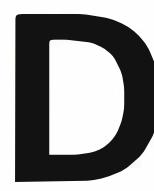

ès la fin du XIXe siècle, la transplantation pancréatique fut proposée comme traitement du diabète [1], mais on a dû attendre les progrès techniques de la dernière décennie pour voir baisser la mortalité après une telle intervention [2]. Actuellement, la greffe pancréatique semble permettre la normalisation à long terme de la glycémie chez le patient diabétique et, par conséquent, l'arrêt de la progression de la plupart des complications chroniques [3, 4]. Mais, malgré l'application d'une immunosuppression continue, $60 \%$ des greffes de pancréas sont rejetées au cours des cinq premières années [2]. C'est pourquoi cette greffe ne se justifie pleinement que chez des patients diabétiques nécessitant une intervention chirurgicale assortie d'une immunosuppression, par exemple une greffe de rein. L'objectif biologique d'une transplantation pancréatique est, dans ce contexte, d'éviter les complications rénales du diabète dans le rein transplanté ; pour le malade, une période d'insulino-indépendance représente évidemment une amélioration de la qualité de vie. La transplantation d'un pancréas chez les autres patients insulinodépendants doit être considérée différemment; en effet, l'intervention aura alors pour but de gưérir la maladie avant que les complications chroniques ne s'installent. Cela nécessite d'intervenir chez des sujets jeunes, au moment où l'on ne peut pas encore prédire dans quelle mesure ils présenteront ou non des complications chroniques. Le succès de la transplantation doit donc être 
comparé à la morbidité et à la mortalité des patients non greffés qui sont traités par l'insuline. Une telle étude comparative n'a, jusqu'à présent, pas été faite et cela pour des raisons évidentes (Tableau I) : l'opération reste complexe; la greffe nécessite une administration continue d'immunosuppresseurs exposant le patient à de sérieux effets secondaires [5] et, enfin, le succès clinique des transplantations pancréatiques isolées est faible $(80 \%$ de rejet en cinq ans [2]).

Théoriquement, plusieurs de ces objections disparaîtraient si la greffe pouvait être réduite au seul tissu nécessaire, c'est-à-dire le tissu endocrine (Tableau I). Le volume à implanter diminuerait d'un facteur 100 , ce qui rendrait l'acte technique plus simple. La dimension des agrégats cellulaires (de 50 à $500 \mu \mathrm{m}$ de diamètre) permettrait une implantation dans des sites où les réactions de rejet sont atténuées. Le volume réduit des greffes permettrait aussi leur stockage en culture ou en cryopréservation, ce qui offrait, d'une part, la possibilité d'un contrôle régulier de qualité et, d'autre part, un éventuel traitement ex vivo qui viserait à réduire leur immunogénicité. Cette dernière modalité permettrait peut-être de se passer d'immunosuppression continue.

A côté des avantages théoriques de l'implant endocrine, il existe un certain nombre d'obstacles et d'inconvénients possibles (Tableau I). Tout d'abord, la préparation de tissu pancréatique endocrine exige des techniques de laboratoire compliquées, qui peuvent entraîner des pertes qualitatives, induisant un contrôle métabolique moins efficace que la greffe d'organe entier, et quantitatives, au point de nécessiter plus d'un pancréas de donneur par receveur. Le caractère ectopique de l'implantation pourrait également diminuer l'efficacité métabolique des cellules transplantées.

De ce fait, contrairement à la transplantation du pancréas, qui a trouvé une application clinique dans les greffes combinées, l'implantation de tissu pancréatique endocrine en est encore à un stade expérimental (Tableau I), sans grand succès chez l'homme [6]. Récemment, cependant, les techni-

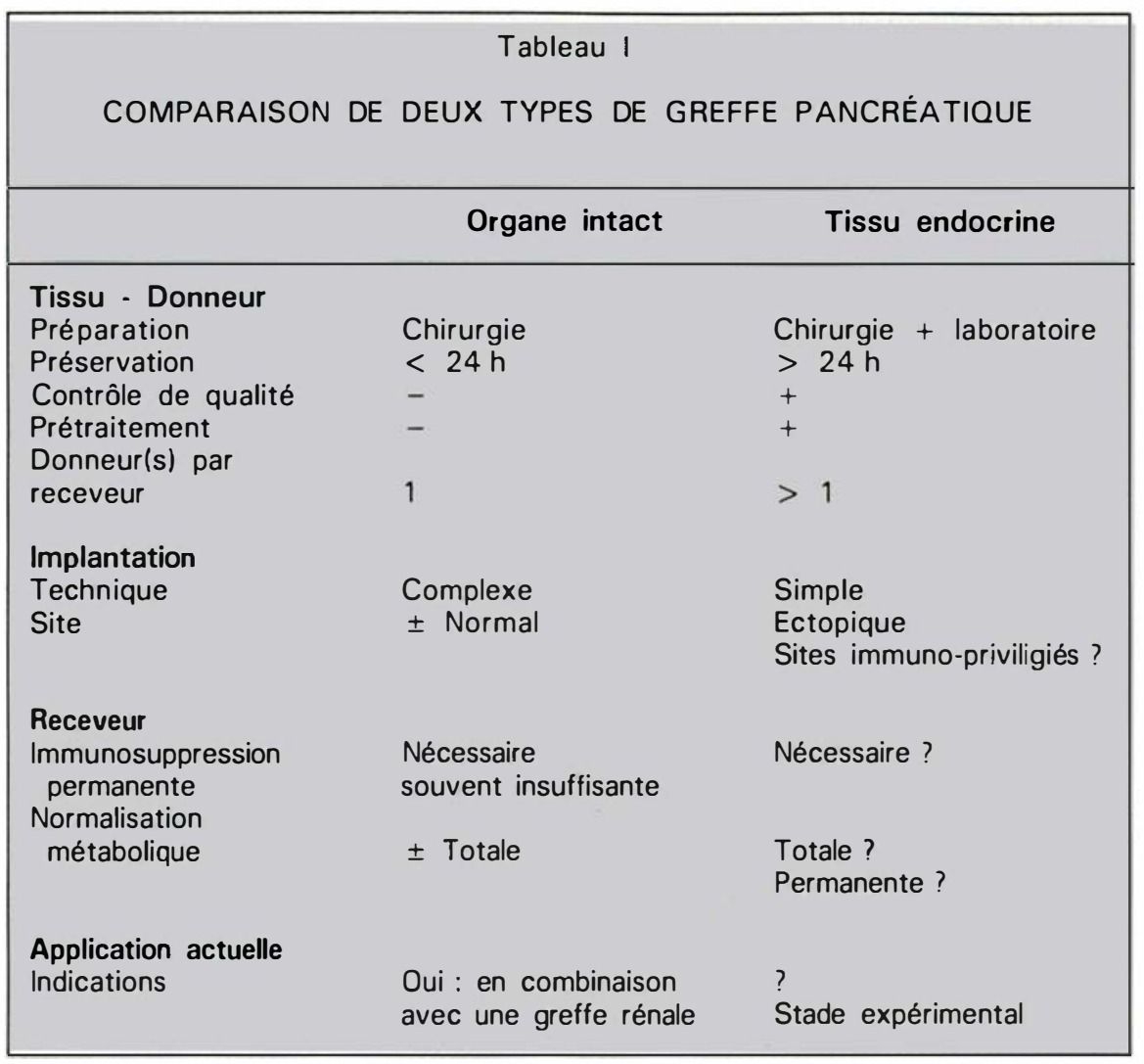

ques d'isolement des îlots humains ont été considérablement améliorées [7-10], permettant de transplanter une plus grande quantité de tissu endocrine. La question reste néanmoins posée de savoir si les greffes insulaires effectuées jusqu'à présent ont été réalisées avec une quantité suffisante de cellules $\beta$ [54]. Dans quelques cas, le tissu endocrine implanté dans le foie [11-13] ou l'épiploon gastrique (Altman et al., communication personnelle) a permis d'équilibrer le diabète. La durée et la qualité de cette équilibration n'étant pas encore connues, on ne peut vraiment comparer à ce jour les résultats des implantations d'îlots et des transplantations de pancréas, ni prévoir l'avenir de la première méthode dans le traitement du diabète. C'est cet avenir que nous nous efforcerons de préciser à la lumière des travaux expérimentaux déjà réalisés (Tableau II, p. 348).

\section{Technique}

\section{- L'isolement}

L'introduction de la digestion par la collagénase [14] a permis d'isoler du tissu endocrine à partir du pancréas, qui est composé, à $98 \%$, d'éléments non endocrines. La méthode a été appliquée à diverses espèces, y compris l'homme. Grâce à l'action enzymatique de la collagénase, les îlots se libèrent du parenchyme exocrine et deviennent ainsi accessibles aux techniques de séparation (Tableau III). Initialement, la séparation des îlots de Langerhans s'effectuait manuellement sous le microscope à dissection. Plus tard, la centrifugation de gradient a permis un isolement à plus grande échelle [8-10, 15] (Tableau III, p. 350). Les préparations obtenues ne peuvent 


\section{Critères}

\section{RÉFÉRENCES}

1. Williams PW. Notes on diabetes treated with extract and by grafts of sheeps pancreas. Br Med J 1894; 1303-4.

2. Sutherland DER, Monday KC, Fryd DS. Results of Pancreas. Transplant Registry. Diabeles 1989; 38 (suppl 1) : 46-54.

3. Land W, Landgraf R, Illner WD, el al. Clinical pancreatic transplantation using the prolamine duct occlusion technique. The Munich experience. Transplantation Proc $1987 ; 19$ (suppl 4) : 75-83.

4. Kennedy WR, Navarro X, Goetz FC, Sutherland DER, Najarian JS. Effects of pancreatic transplantation on diabetic neuropathy. $N$ Engl J Med 1990 ; 322 : 1031-7.

5. Cohen DJ, Loerscher R, Rubin MF, Tilney NL, Carpenter CB, Strom TB. Cyclosporine : a new immunosuppressive agent for organ transplantation. Ann Internal Med 1984; 101 : 667-82.

6. Hering BJ, Bretzel RG, Federlin K. Current status of clinical islet transplantation. Horm Melabol Res 1988 ; 20 : 537-45.

7. Gray DWR, McShane P, Grant A, Morris PJ. A method for isolation of islets of Langerhans from the human pancreas. Diabeles 1984 ; 33 : 1055-60.

8. Rajotte RV, Warnock GL, Evans MG, Ellis D, Dawidson I. Isolation of viable islets of Langerhans from collagenaseperfused canine and human pancreata. Transplantation Proc 1987 ; 19 : 918-22.

9. Ricordi C, Lacy PE, Finke EH, Olack BJ, Scharp DW. Automated method for isolation of human pancreatic islets. Diabetes $1988 ; 37: 413-20$

10. Alejandro R, Mintz DH, Noel J, el al. Islet cell transplantation in type 1 diabetes mellitus. Transplant Proc 1987 ; 19 : 2359-61.

11. Sharp DW, Lacy PE, Santiago JV, el al. Insulin independence after islet transplantation into type 1 diabetic patient. Diabetes $1990 ; 329$ : 515-8.

12. Tzakis AG, Ricordi C, Alejandro R, $e l$ al. Pancreatic islet transplantation after upper abdominal exenteration and liver replacement. Lancel 1990 ; 336 : 402-5.

13. Warnock GL, Kneteman NM, Ryan EA, el al. Continued function of pancreatic islets after transplantation in type 1 diabe- cependant pas être considérées comme purement endocrines: des agrégats de cellules exocrines et des cellules des canaux pancréatiques (cellules ductales) restent attachés tandis qu'à l'intéricur même de l'îlot, on reconnaît des cellules endothéliales, des terminaisons nerveuses, des fibroblastes, des macrophages, des cellules dendritiques et des cellules sanguines (figure 1). Peu de travaux mentionnent les pourcentages respectifs de ces cellules non endocrines. Leur nombre peut varier sclon l'expérience, la méthode utiliséc et l'espèce étudiéc. Cette variabilité disparaît si l'on prépare des cellules $\beta$ pures. La technique de purification des cellules productrices d'insuline a été développée chez le rat mais s'applique aussi à d'autres espèces. Elle consiste en une dissociation du tissu insulaire suivie d'un tri de cellules sur la base de leur autofluorescence (Tableau III, [16]). Les cellules $\beta$ purifiées peuvent être regroupées en particules ayant un diamètre de 50 à $200 \mu \mathrm{m}$ (figure 2, p. 351, [17]).

La caractérisation des préparations insulaires est limitéc par le manque de données quantitatives sur le rendement des techniques utilisées. Le nombre d'îlots donne en effet peu d'information si on ne le compare pas au volume de tissu endocrine de départ. Or, le rendement en cellules $\beta$ dépend probablement de plusieurs paramètres mal maîtrisés, d'autant plus que l'analyse des préparations d'îlots jusqu'à présent utilisées a été insuffisante. Cette insuffisance, liée au désir de l'expérimentateur de transplanter rapidement le matéricl isolé, a empêché jusqu'ici d'établir une claire corrélation entre la qualité et la quantité de cellules $\beta$ transplantées et les effets métaboliques obtenus.

\section{- L'implantation}

Des préparations de tissu endocrinc pancréatique de taille et de pureté différentes ont été implantées dans divers sites (Tableau IV, p. 352). La plupart de ces expériences ont été conduites chez le rat ou la souris, rendus diabétiques par la streptozotocine. Ainsi a-t-on constaté que des implantations dans le foie, la capsulc rénale, la rate, le péritoine, le testicule, la peau, le thymus et le cerveau étaient techniquement aisées [18]. Chez les animaux de plus grande taille, tels que le chien et le singe, la plupart des greffes ont été effectuées dans le foie ou la rate $[18,19]$. Rappclons que, chez l'homme, le foie et l'épiploon gastrique ont été choisis avec succès [11-13].

A l'heure actuelle, on peut donc affirmer que l'implantation de tissu pancréatique endocrine isolé est techniquement faisable. La méthode offre l'avantage de pouvoir s'effectuer dans des sites différents. Ce choix peut, tout comme celui du tissu donneur, représenter un instrument important 


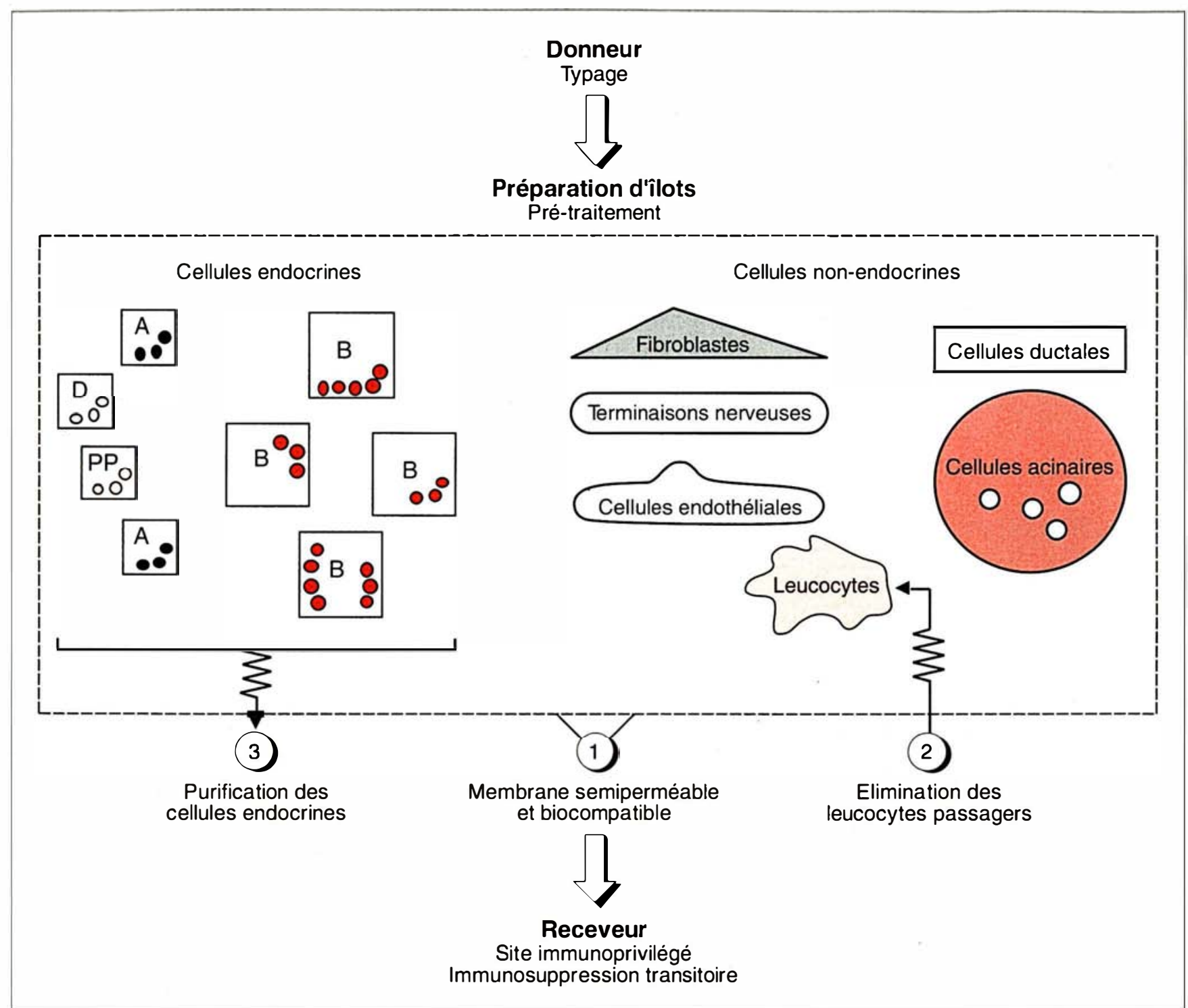

Figure 1. Préparation de greffes insulaires. Une digestion à la collagénase permet l'isolement du tissu insulaire des organes pancréatiques. Les préparations isolées contiennent plusieurs types cellulaires, de nature endocrine ou nonendocrine. Parmi les cellules endocrines, on distingue des cellules à glucagon (A), à insuline (B), à somatostatine (D) et au polypeptide pancréatique (PP). II existe différents moyens d'améliorer la tolérance immunologique des rongeurs aux allogreffes insulaires. Une première méthode se situe au niveau de la sélection des donneurs : un avantage immunologique a été démontré pour des préparations insulaires provenant de multiples donneurs qui diffèrent dans leur typage du complexe majeur d'histocomptabilité [53]. Une deuxième approche consiste dans le prétraitement des préparations insulaires. Trois formes de prétraitement prolongent la survie des allogreffes : (1) l'enveloppement de tissu par une membrane semi-perméable et biocompatible; (2) l'élimination des leucocytes passagers; (3) la purification des cellules endocrines. Le troisième niveau d'intervention se situe au niveau du receveur et vise l'implantation dans un site immunoprivilégié et/ou l'immunosuppression pharmacologique. 


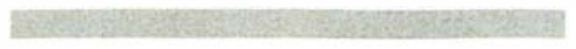
RÉFÉRENCES

14. Lacy PE, Kostianovsky MK. Method for the isolation of intact islets of Langerhans from the rat pancreas. Diabctes 1967 : $16: 35-9$.

15. Lindall A, Steffes $M$, Sorenson R. Immunoassayable insulin content of subcellular fractions of rat islets. Endocrinology $1969 ; 85: 218-23$.

16. Pipeleers D. In't Veld P, Van De Winkel M, Maes E, Schuit FC, Gepts W. A new in vitro model for the study of pancreatic A and B cells. Endocrinology 1985; 117 : 806-16

17. Pipeleers D, Schuit F, In't Veld $P$, et al. Interplay of nutrients and hormones in the regulation of insulin release. Endocrino$\log y 1985 ; 117: 824-33$

18. Gray DWR, Morris PJ. Transplantation of isolated pancreatic islets. In : Groth CG, ed. Pancreatic Transplantation. Philadelphia : Saunders, $1988: 363-90$

19. Nason RW, Rajotte RV, Warnock GL Pancreatic islet cell transplantation: past, present and future. Diabeles Res 1988 ; $T$ : $1-11$.

20. Tze IVJ, Tai J. Immunological studies in diabetic rat recipients with a pancreatic islet cell allograft in the brain. Transplanta tion $1989 ; 47: 1053-7$.

21. Gray DWR. The role of exocrine tissue in pancreatic islet transplantation. Trans plant Int 1989 ; 2 : $41-5$.

22. Gray DWR, Morris PJ. Cyclosporine and pancreas transplantation. World J Surg $1984 ; 8: 230-5$.

23. Gill RG, Lafferty $\mathrm{KJ}$. The role of islet transplantation in the treatment of insulindependent diabetes mellitus. Transplantation Immunol 1989 ; 9 : 165-86.

24. Lacy PE. Perspectives of islet cell transplantation in diabetes. In : Lefèbvre PJ, Pipeleers DG, eds. The Pathology of the Endo crine Pancreas in Diabeles. Berlin-Heidelberg : Springer, 1988 : 310-28.

25. Reach G. Artifical or bioartifical systems for the totally automatic treatmen of diabetes mellitus : the gap between the dream and the reality. Diab Nutr Metab 1989 ; 2 : 165-70

26. O'Shea GM, Goosen MFA, Sun AM. Prolonged survival of transplanted islets of Langerhans encapsulated in a biocompatible membrane. Biochim Biophys Acla 1984 ; 804: 133-6.

27. O'Shea GM, Sun AM. Encapsulation of rat islets prolongs xenograft survival in diabetic mice. Diabetes $1986 ; 35$ : 943-6.

\begin{tabular}{|c|c|c|}
\hline \multicolumn{3}{|c|}{$\begin{array}{c}\text { Tableau III } \\
\text { L'ISOLEMENT DE TISSU ENDOCRINE PANCRÉATIQUE }\end{array}$} \\
\hline & $\begin{array}{l}\text { Rendement* } \\
\text { en cellules } \beta \\
(\%)\end{array}$ & $\begin{array}{l}\text { Pureté } \\
\text { en cellules } \beta \\
(\%)\end{array}$ \\
\hline collagénase | & 100 & $<2$ \\
\hline $\begin{array}{l}\quad \begin{array}{c}\text { Fragments } \\
\text { tissulaires } \\
\text { manuel }\end{array} \\
\text { centrifugation } \\
\text { en gradient }\end{array}$ & $>70$ & $<2$ \\
\hline $\begin{array}{l}\begin{array}{c}\text { Préparation } \\
\text { d'îlots }\end{array} \\
\text { dissociation | } \\
\text { auto-FACS }\end{array}$ & $<70$ & $20-80$ \\
\hline Cellules $\beta$ & $<50$ & $>90$ \\
\hline
\end{tabular}

* valeurs déterminées dans notre laboratoire pour le pancréas de rat, de porc et humain. Tri de cellules par autofluorescence-activated cell sorting (auto-FACS).

pour la réalisation des deux étapes suivantes, la tolérance biologique et la qualité du contrôle métabolique (Tableau IV).

\section{Tolérance biologique}

L'implant doit pouvoir vivre en symbiose avec les tissus du receveur. Il faut donc déterminer si l'implantation suscite des effets secondaires chez l'hôte et si l'hôte tolère la greffe.

\section{- Les effets secondaires chez l'hôte}

Il y a peu de données concernant les lésions provoquées par des îlots pancréatiques dans un environnement ectopique. L'injection dans la veine porte provoque une hypertension portale transitoire [18]. Dans le foie, à longue échéance, les cellules endocrines établissent des contacts directs avec les hépatocytes qui se chargent en glycogène et en lipides [19]. Théoriquement, les implantations intracérébrales pourraient provoquer une compression du tissu cérébral, mais ccla n'a jamais été rapporté chez l'animal d'expérience [20].

Certains effets secondaires pourraient aussi être provoqués par des produits de sécrétion de la greffe, endocrines ou exocrines, dans ce dernier cas dus à la contamination du matériel insulaire par du matériel pancréatique exocrine. Une telle contamination peut entraîner une coagulation intravasculaire [21], encore accélérée par la présence de débris et de tissus nécrotiques.

\section{- La survie de l'implant}

Les réactions de rejet du tissu insulaire histo-incompatible n'ont pu, jusqu'à présent, être maîtrisées par la seule immunosuppression pharmacologique du receveur [22], mais elles pourraient cependant être affaiblies par un prétraitement du tissu donneur ou par son implantation dans des sites dits "immunoprivilégiés" (par exemple : testicule, cerveau ct thymus ; m/s $n^{\circ} 10$, vol. 6, p. 1014). 


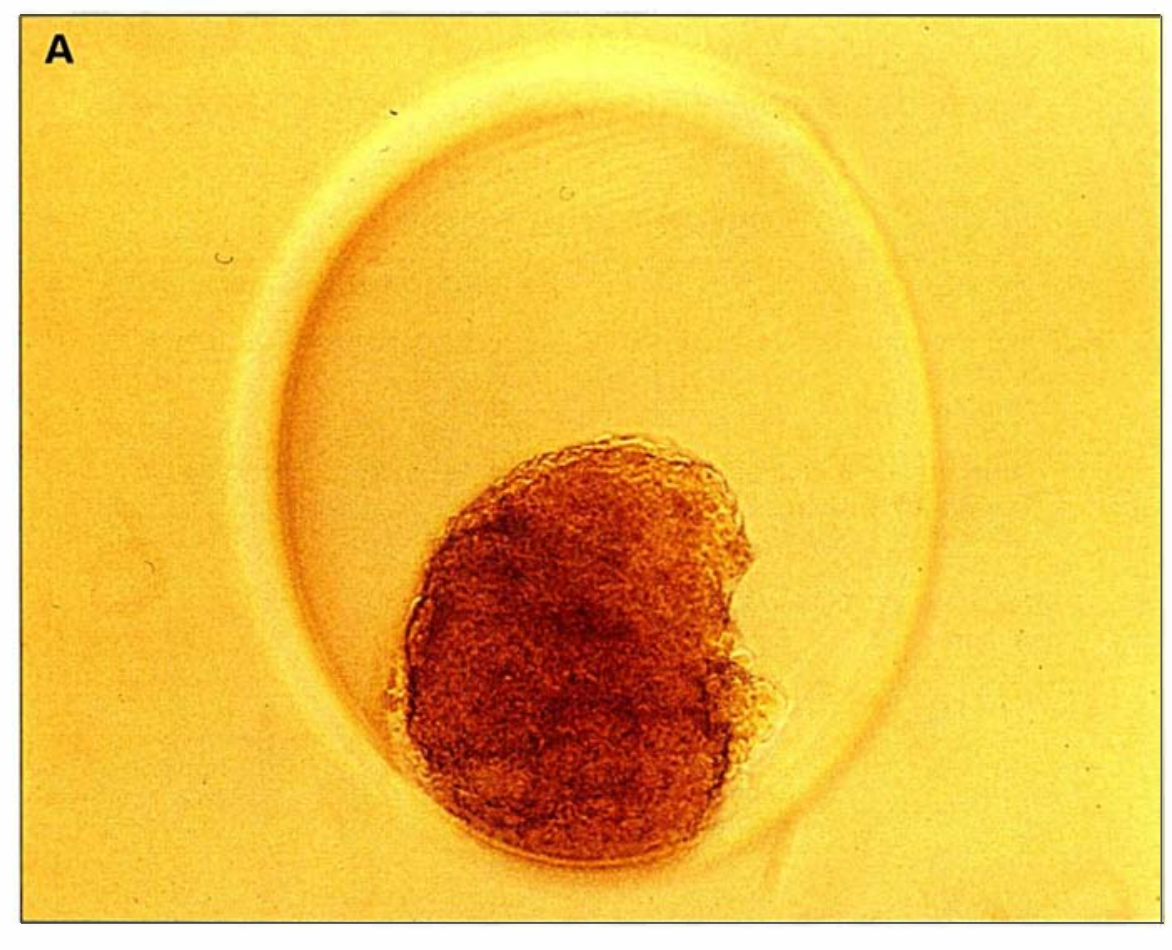

\section{B}

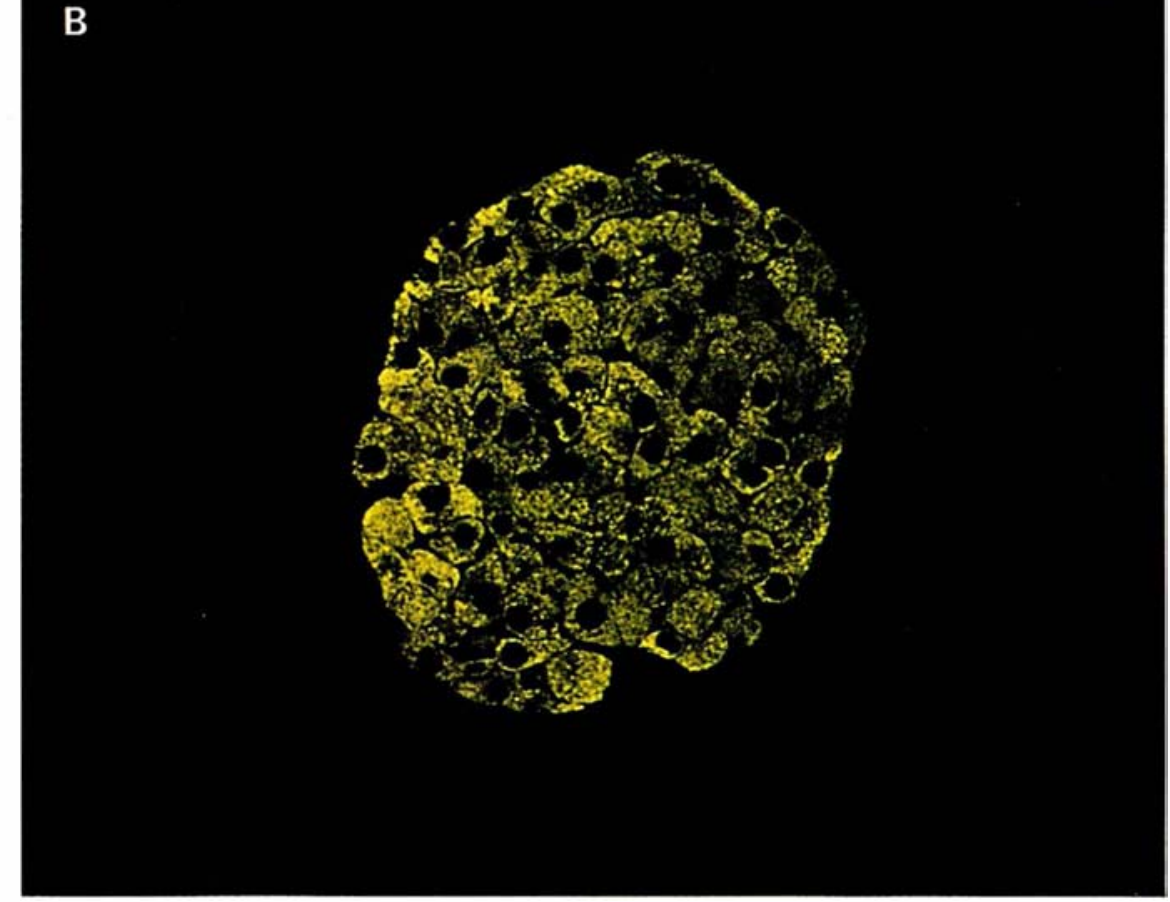

Figure 2. Préparations insulaires ayant subi un prétraitement visant la diminution de l'immunogénicité tissulaire. (A) llot de rat encapsulé par une membrane d'alginate-polylysine (document fourni par le Dr Foriers, de notre université. (B) agrégat de cellules $\beta$ purifiées (immunofluorescence en fluorescéine après réaction avec un anticorps anti-insuline).
- Les pré-traitements du tissu donneur

Destinés à affaiblir son immunogénicité chez un receveur histoincompatible, ils sont de plusicurs types, décrits dans plusieurs articles de revue récents [18, 23, 24, 27, 28].

- La membrane semi-perméable et biocompatible enveloppant le tissu insulaire. Il s'agit là d'une idéc séduisante qui a pour but de limiter la communication entre les tissus du receveur et le tissu transplanté aux petites molécules, comme le glucose et l'insuline, tandis que des substances plus volumineuses comme les immunoglobulines seraient exclues, de même, évidemment, que les cellules (figure 1). Ce principe offre des perspectives pour les allogreffes aussi bien que pour les xénogreffes [29]. Plusieurs possibilités techniques ont été décrites pour envelopper les îlots par des membranes semi-perméables [25]. I.a capsule d'alginate-polylysine (figure 2) a été utiliséc avec succès pour des allo- et xéno-transplantations dans le péritoine de rongeurs diabétiques [26, 27]. Des vaisseaux semi-perméables ont servi à l'implantation de cellules humaines chez des animaux de laboratoire [28]. Malheureusement, plusicurs études ont noté une perte progressive de la fonction endocrine, v'raisemblablement à cause de réactions inflammatoires autour de l'implant [25]. Les membranes semiperméables risquent donc de perdre lcurs propriétés intrinsèques à cause d'une biocompatibilité insuflisante. De futurs développements devront aussi quantificr la survic à long terme des cellules $\beta$ greffées dans unc capsule immunoprotectrice.

- Les destructions des leucocytes passagers dans le tissu donneur. Cettc approche est fondéc sur l'idéc que les leucocytes passagers présents dans le tissu greffé joucnt un rôle important dans le déclenchement des réactions de rejet $[18,23,24]$. Les cellules dendritiques, avec leur fortc expression des antigènes de classe II du complexe majcur d'histocompatibilité, sont considérées comme étant les plus immunogènes. Les leucocytes passagers peuvent être détruits par culture à $24^{\circ}$ ou à $37^{\circ} \mathrm{C}$, par exposition à la lumière UV, par incubation à $95 \%$ d'oxygène ou par exposition au complément après mise en présence 


\section{RÉFÉRENCES}

28. Altman JJ, Houlbert D, Callard P, et al. Long-term plasma glucose normalization in experimental diabetic rats with macroencapsulated implants of benign human insulinoma. Diabeles 1986 ; $35: 625-33$.

29. Reach G, Jafrin MY. Les pancréas bioartificiels. médecine/sciences $1986 ; 2$ : 87-93.

30. Pipeleers D, Pipeleers-Marichal $\mathbf{M}$, Hannaert JC, et al. Transplantation of purified islet cells in diabetic rats. 1. Standartization of islet cell grafts. Diabeles (sous presse).

31. Pipeleers D, Pipeleers-Marichal $M$, Vanbrabandt B, Duys S. Transplantation of purified islet cells in diabetic rats. 2. Immunogenicity of allografted islet B cells. Diabetes 1990 (sous presse).

32. Pipeleers-Marichal M, Ling ZD, Teng $\mathrm{H}$, Pipcleers D. Transplantation of purified islet cells in diabetic rats. 3. Immunosuppressive effect of cyclosporin. Diabetes 1990 (sous presse).

33. Lacy PE, Davie JM, Finke EH. Prolongation of islet xenograft survival (rat to mouse). Diabctes 1981 ; 30 : 285-91.

34. Posselt AM, Backer CF, Tomaszewski JE, Markwann JF, Choti MA, Naji A. Induction of donor-specific unresponsiveness by intrathymic islet transplantation. Science $1990 ; 249: 1293-5$.

35. Eisenbarth G. Type 1 diabetes mellitus. A chronic autoimmune disease. $N$ Engl J Med 1986 ; 314 : 1360-8

36. Sibley RK, Sutherland DER, Goetz F, Michael AF. Recurrent diabetes mellitus in the pancreas iso- and allograft. A light and electron microscopic and immunohistochemical analysis of four cases. Laboralory Invest $1985 ; 53: 132-44$

37. Fan MT, Lum ZP, Fu XW, Levesque L, Tai IT, Sun AM. Reversal of diabetes in BB rats by transplantation of encapsulated pancreatic islets. Diabeles $1990 ; 39$ : 519-22.

38. Calafiore R, Calcinaro F, Basta G, et al. A method for the massive separation of highly purified adult porcine islets of Langerhans. Melabolism 1990; 39: 175-81.

39. Woehrle M, Markmann JF, Silvers WK, Barker CF, Naji A. Transplantation of cultured pancreatic islets to BB rats. Surgery $1986 ; 100: 334-41$.

40. Chabot JA, Lau H, Reemtsma K, Hardy MA. Long-term survival of islet allografts in spontaneously diabetic BB rats without chronic immunosuppression. Trans-

Tableau IV

\section{LA MISE EN OEUVRE DE LA TRANSPLANTATION CELLULAIRE}

\begin{tabular}{|c|c|c|}
\hline \multicolumn{3}{|c|}{ Variables expérimentales } \\
\hline \multicolumn{2}{|c|}{ Tissu donneur } & Site d'implantation \\
\hline \multicolumn{2}{|c|}{$\begin{array}{l}\text { Génotype } \\
\text { Composition cellulaire } \\
\text { Nombre de cellules } \beta \\
\text { Types et nombre } \\
\text { de cellules non } \beta \\
\text { Prétraitement et forme } \\
\text { des agrégats }\end{array}$} & $\begin{array}{l}\text { Foie, capsule rénale } \\
\text { épiploon, péritoine } \\
\text { rate, thymus } \\
\text { cerveau }\end{array}$ \\
\hline \multicolumn{3}{|c|}{ Greffe optimalisée } \\
\hline \multicolumn{3}{|c|}{ Receveur } \\
\hline \multirow[t]{2}{*}{ But } & Métabolique & Immunologique \\
\hline & $\begin{array}{l}\text { Correction pe } \\
\text { évitant l'appa } \\
\text { de lésions ch }\end{array}$ & $\begin{array}{l}\text { Survie des allogreffes } \\
\text { sans immunosuppression }\end{array}$ \\
\hline
\end{tabular}

avec des anticorps dirigés contre les antigènes membranaires des leucocytes. Quand le tissu insulaire subit l'un de ces prétraitements ex vivo, ses chances de survie après allogreffe augmentent considérablement, au moins chez des rongeurs diabétiques [18, 23, 24], figure 1). Il reste à démontrer que ces techniques, qui visent à éliminer un seul type cellulaire, ont une action suffisamment spécifique pour ne pas exercer d'action destructive sur d'autres types cellulaires. Par ailleurs, il n'est pas prouvé que le succès obtenu chez les animaux de laboratoire puisse être extrapolé à d'autres espèces, surtout si la méthode se fonde sur des facteurs aussi variables que l'expression d'antigènes à la surface des membranes cellulaires. Finalement, dans cette approche, on ne tient pas compte de l'existence éventuelle de cellules non leucocytaires comme facteur d'immunogénicité de la greffe.
Ces prétraitements constituent donc des méthodes potentiellement intéressantes dont il convient encore de tester l'efficacité et les limites.

- La purification des cellules pancréatiques endocrines. Les techniques de purification des cellules $\beta$ (figure 2) et des autres cellules endocrines et non endocrines du pancréas $[16,30]$ ont permis d'évaluer dans quelle mesure chacun de ces groupes cellulaires participe à l'immunogénicité des greffes insulaires [31]. Ces études ont démontré que, bien qu'immunogènes après une allotransplantation, les cellules $\beta$ purifiées provoquent une réaction de rejet moins importante et moins aiguë que les implants d'îlots isolés [31, 32] (figure 3). Les cellules non endocrines et les débris cellulaires accroissent la vitesse et l'intensité de la réaction cytotoxique [32] alors que la présence d'autres cellules endocrines, telles les cellules A, augmentent la survie des 
cellules $\beta$ allogreffées [31]. La même étude a confirmé l'exacerbation du phénomène de rejet en présence de tissu exocrine $[22,32]$. L'implantation de greffes d'une composition cellulaire bien déterminée constitue donc une méthode puissante pour produire des greffes de basse immunogénicité (figure 1). Il reste à déterminer si cette approche est également valable pour d'autres espèces que le rat. Un essai chez l'homme semble justifié mais exigera un certain nombre d'adaptations techniques permettant la purification de cellules $\beta$ à grande échelle.

- Les sites priviligiés

Le petit volume que représente le pancréas endocrine rend possible l'implantation en divers sites et la comparaison des temps de survie de ces allogreffes (Tableau IV). Ces études ont été effectuées chez des rongeurs et ont permis d'identifier une série de sites dits "priviligiés " du point de vue immunologique. Ainsi l'on a pu constater une survie permanente d'allo- et de xénogreffes implantées dans le testicule [33] ; la greffe intrathécale fournirait également une protection absolue aux îlots allotransplantés [2], et il en serait de même pour le thymus $\left(\mathrm{m} / \mathrm{s} n^{\circ} 10\right.$, vol. 6, p. 1014) [34]. En outre, l'implantation dans le thymus présenterait l'avantage de conduire à une tolérance permanente pour des îlots de génotypes identiques [34]. Bien que plusieurs questions restent à examiner, le choix d'un site limitant la réaction immunologique est certainement un élément important de la réussite des greffes insulaires.

\section{- Les réactions auto-immunes}

Le diabète insulinodépendant semble être le résultat d'une destruction auto-immune des cellules $\beta$ du pancréas [35]. Cette autoréactivité contre les cellules $\beta$ pourrait également interférer avec la survie des cellules $\beta$ implantées [36], ce qui assombrit l'avenir de la transplantation chez le diabétique. Dans le cas d'une autoréactivité restreinte au complexe majeur d'histocompatibilité (CMH), des cellules $\beta$ provenant d'un donneur ayant un CMH différent de celui du receveur ne seraient pas cibles de l'attaque auto-immune, mais il faudrait alors éviter la destruction de la greffe par l'alloréactivité de l'hôte. Des méthodes permet- tant d'empêcher le rejet d'allogreffes insulaires chez des rats rendus diabétiques à la streptozotocine ont été décrites précédemment dans cet article. Plusieurs de ces méthodes ont également été appliquées avec succès aux animaux souffrant d'un diabète auto-immun. Des allo- et xénogreffes encapsulées guérissent le diabète de rats $\mathrm{BB}$ et de souris $\operatorname{NOD}[37,38]$. Des îlots prétraités $[39,40]$ ou des cellules endocrines purifiées (D. Pipeleers, sous presse) normalisent la glycémie de façon permanente chez les rats $\mathrm{BB}$ diabétiques, dans la mesure où le donneur et le receveur ont bien des CMH différents. Des implantations intratesticulaires ou intracérébrales garantissent également chez le rat $\mathrm{BB}$ une immunoprotection [41, 42].

Nous pouvons donc conclure que, chez l'animal, des antécédents de destruction auto-immune du pancréas n'excluent pas que puisse être réalisée avec succès une greffe de cellules $\beta$ capables de corriger à long terme le métabolisme glucidique.

\section{Le contrôle métabolique}

Depuis quinze ans, il est établi que la transplantation d'îlots isolés est capable de normaliser des paramètres biologiques perturbés chez les animaux diabétiques de laboratoire [19, $43,44]$. Cet effet est également obtenu après injection d'insuline dont on connaît depuis longtemps l'effet normalisateur sur la glycémie de base et la glycosurie. L'intérêt des greffes insulinoproductrices est qu'elles garantissent une homéostasie glucidique plus physiologique qui pourrait empêcher le développement de lésions chroniques au niveau des tissus vasculaires, nerveux, rénaux et oculaires. Pour démontrer la qualité du contrôle métabolique, il sera nécessaire de suivre à long terme la tolérance au glucose ainsi que le métabolisme périphérique des animaux transplantés, et de comparer les résultats à ceux obtenus par l'insulinothérapie.

\section{- La tolérance au glucose}

Relativement peu d'études ont été consacrées au métabolisme du glucose chez les receveurs de tissu insulaire.

Des normalisations de la tolérance au glucose ont été rapportées chez le rat [44], mais on ne sait pas encore clairement si la correction est complète et permanente $[18,44,45]$. Nous avons constaté que le contrôle par des cellules transplantées disparaissait en cas de stress, comme si l'absence d'innervation des greffes limitait leur sécrétion d'insuline dans certaines circonstances [44]. Le même phénomène pourrait aussi expliquer l'absence de la phase céphalique dans le processus sécrétoire [46]. En l'absence de données précises sur le nombre des cellules $\beta$ transplantées et survivantes, il n'est pas encore possible de déterminer l'importance de la masse cellulaire à la fois nécessaire et suffisante à un bon contrôle de la glycémie. Cette étude devra tenir compte d'éventuelles différences selon le site d'implantation et l'âge du receveur. Lorsque ces informations auront été obtenues et que l'on saura disposer de préparations cellulaires bien caractérisées, nous pensons qu'il sera possible de standardiser la réalisation de greffes aptes à assurer leur fonction dans le nouvel environnement qui leur est imposé [47].

Chez le chien, aussi bien que chez le singe pancréatectomisé, une normalisation de la glycémie de base a été obtenue par une autotransplantation de fragments insulaires $[19,48,49]$. $\mathrm{La}$ correction métabolique s'est cependant affaiblie au cours du temps, indiquant une mort ou une insuffisance cellulaire prématurée. Le manque de données sur les caractéristiques initiales des greffons complique toute interprétation de ce phénomène.

\section{- Les lésions chroniques}

Nous ne disposons que de peu d'informations concernant l'effet de la transplantation insulaire sur les complications chroniques du diabète. La plupart des données proviennent d'expériences chez les rats ou les souris traités par la streptozotocine. Ces animaux développent des lésions rénales comparables à celles constatées chez les patients diabétiques insulinodépendants ; il faut cependant tenir compte également de l'effet néphrotoxique de l'agent diabétogène utilisé dans le modèle expérimental. L'épaississement de la membrane basale et l'expansion du mésangium peuvent être utilisés 


\section{RÉFÉRENCES}

41. Tze WJ, Tai J. Successful intracerebral allotransplantation of pancreatic endocrine cells in spontaneous diabetic BB rats without immunosuppression. Metabolism 1984; 33 785-9.

42. Woehrle MF, Markmann JF, Armstrong J, Naji A. Effect of transplant site on islet allograft survival in BB rats. Transplantation Proc 1987 ; 19 : 925-7.

43. Kemp CB, Knight MJ, Scharp DW, Lacy PE, Ballinger WF. Transplantation of isolated pancreatic islets into the portal vein of diabetic rats. Nature $1973 ; 244: 447$

44. Pipeleers D, Pipeleers-Marichal M, Karl I, Kipnis D. Secretory capability of islets transplanted intraportally in the diabetic rat. Diabetes $1978 ; 27: 817-24$

45. Hegre OD. Islet cell transplantation. In : Volk BW, Arquilla ER, eds. The Diabetic Pancreas. Plenum Medical, 1985 513-42.

46. Louis-Sylvestre J. Feeding and metabolic patterns in rats with troncular vagotomy or with transplanted $\beta$-cells. Am J Physiol 1978 ; 235 : E119.

47. Pipeleers D. The biology of pancreatic B-cells (Minkowski Lecture). Diabetologia $1987 ; 30: 277-91$.

48. Morris PJ, Gray DW, Sutton R. Pancreatic islet transplantation. British Medical Bulletin 1989 ; 45 : 224-41.

49. Alejandro R, Cutfield RG, Shienvold FL, et al. Natural history of intrahepatic canine islet cell autografts. J Clin Invest $1986 ; 78: 1339-48$.

50. Orloff MJ, Macedo C, Macedo A, Greenleaf GE. Comparison of whole pancreas and pancreatic islet transplantation in controlling nephropathy and metabolic disorders of diabetes. Ann Surg 1987; 206 : 324-34.

51. Hoffman L, Mandel TE, Carter WM, et al. A comparison between islet transplantation and parenteral insulin in the control of diabetes and prevention of renal complications in mice. Metabolism 1983; 32 : 451-6.

52. Steffes MW, Brown DM, Basgen J, Mauer SM. Amelioration of mesangial volume and surface alterations following islet transplantation in diabetic rats. Diabetes $1980 ; 29: 509-15$.

53. Kanai T, Porter J, Monaco AP, Maki T. Successful treatment of experimental diabetes by sequential transplantations of multiple-donor pancreatic islet allografts. Transplantation $1989 ; 47$ : 3-6.

54. Weir GC, Bonner-Weir S, Leahy JL. Islet mass and function in diabetes and


Figure 3. Effet de la purification cellulaire sur l'immunogénicité du tissu pancréatique allogreffé chez le rat diabétique. (A) Infiltration massive des sites d'implantation, 9 jours après l'injection d'îlots isolés. Le foie ne contient plus de tissu endocrine et le receveur est redevenu diabétique (réaction hématoxyline-éosine). (B) Infiltration minime à la périphérie d'un agrégat de cellules $\beta$ purifiées. Le foie présente de multiples agrégats endocrines et le receveur est resté normoglycémique pendant plus de 10 semaines (réaction hématoxyline-éosine). 
comme des indices des effets périphériques de l'hyperglycémie chronique. Ces lésions ne se développent pas chez des animaux dont le diabète a été corrigé par une greffe pancréatique [50]. Deux études à court terme ( $\leqslant 6$ mois) ont démontré leur absence chez des rats portant des îlots intraportaux, mais d'autres articles n'ont pas confirmé cette observation [51, 52]. Dans une étude à long terme (deux ans), Orloff et al. constatent que les lésions rénales sont aussi prononcées chez les rats transplantés que chez les rats témoins diabétiques [50]. Ce phénomène s'expliquerait par une incapacité des greffes à normaliser durablement les glycémies.

Cette étude nécessite d'autant plus une confirmation que les données restent imprécises quant à la quantité et à la qualité des îlots injectés, ainsi que pour leur survie après transplantation.

Nous ne disposons pas à l'heure actuelle de suffisamment de données démontrant que les greffes insulaires évitent le développement des complications chroniques du diabète. Plusieurs données indiquent que les cellules $\beta$ transplantées n'achèvent pas toujours le même degré de tolérance au glucose que le pancréas endocrine intact. Jusqu'à présent, relativement peu d'attention a été consacrée au contrôle métabolique des greffes d'îlots. Davantage de travail expérimental sera nécessaire pour clarifier ce critère essentiel.

\section{Conclusion}

En conclusion, des progrès importants ont été faits dans la préparation des îlots de Langerhans ou des cellules $\beta$ isolées, dans leur greffe dans des sites minimisant le rejet immun et dans leur prétraitement diminuant leur immunogénicité pour l'hôte. Une correction, au moins à court terme, des désordres de la glycorégulation a pu être obtenue dans de nombreux cas. Des incertitudes demeurent cependant quant à la fonction à long terme de ces implants endocrines, à la qualité du contrôle métabolique et à la prévention des lésions chroniques du diabète

\section{Summary}

Implantation of pancreatic endocrine tissue

Combined kidney and pancreas transplantation has gained technical and metabolic success in diabetic patients. Single pancreas grafting is, however, not applicable as a cure for patients without chronic complications. Implantation of islet tissue can be considered as alternative if it succeeds in long-term metabolic normalization without the need for permanent immunosuppression. Recent reports indicate the technical feasibility of islet transplantation in man. The issues of biologic tolerance and metabolic control can so far solely be analysed in animal models. Serious side effects were noticed in recipients of grafts which were massively contaminated by exocrine tissue. Isogenic islets survived in different sites, but allogenic tissue was rapidly destroyed except when it was implanted in testis, thymus or brain. The alloreactivity of the host could be reduced by pretreating the donor tissue. Three forms of pretreatment resulted in longterm survival of islet cell allografts : encapsulation by a semipermeable and biocompatible membrane, destruction of passenger leucocytes and purification of endocrine islet cells. Surviving islet grafts were characterized by a basal normoglycemia and, in some studies, by normalized glucose tolerance curves. Other reports indicate that metabolic control is not completely and/or permanently restored by islet transplantation, and may therefore fail to prevent the development of chronic complications. Future research should assess whether islet cell grafts can completely correct the diabetic state.

\section{TIRÉS A PART}

